\title{
Improving Virtual Endoscopy for the Intestinal Tract
}

\author{
Matthias Harders ${ }^{1}$, Simon Wildermuth ${ }^{2}$, \\ Dominik Weishaupt ${ }^{2}$, and Gábor Székely ${ }^{1}$ \\ 1 Swiss Federal Institute of Technology \\ Communication Technology Laboratory \\ ETH Zentrum, CH-8092 Zürich, Switzerland \\ 2 University Hospital Zurich \\ Institute of Diagnostic Radiology \\ Raemistrasse 100, CH-8091 Zürich, Switzerland \\ \{mharders, szekely\}@vision.ee.ethz.ch, \\ \{dominik.weishaupt, simon.wildermuth\}@dmr.usz.ch
}

\begin{abstract}
We present a system that opens the way to apply virtual endoscopy on the small intestines. A high-quality image acquisition technique based on MR as well as a haptically assisted interactive segmentation tool was developed. The system was used to generate a topologically correct model of the small intestines. The influence of haptic interaction on the efficiency of centerline definition has been demonstrated by a user study.
\end{abstract}

\section{Introduction}

The importance of radiologic imaging in the diagnosis of diseases in the intestinal tract has increased dramatically in recent years. One precursor of this development is virtual colonoscopy, which represents a promising method for colorectal cancer screening. In the early 1990s, Vining et al. [10] were the first to report on the technical feasibility of virtual colonoscopy simulating conventional endoscopic examinations. Its advantage is increased patient comfort, due to noninvasiveness, reduced cost as well as reduced sedation time. Results from recent studies $[1,7]$ show the accuracy to be comparable to conventional colonoscopy for detection of polyps of significant size.

Nevertheless, virtual endoscopic evaluation of the intestines has so far been limited to the colon, but several diseases exist that also necessitate a radiologic exam of the small intestines - especially, since the small bowel can not be assessed completely by conventional methods. Virtual endoscopy of the small intestines is much more difficult then virtual colonoscopy because the tubular structure often follows a tortuous and curved path through $3 \mathrm{D}$ space. This makes the accurate tracing of the geometry an extremely difficult task. Furthermore, the tightly folded structure is often sliced at an oblique angle, resulting in extreme deterioration of image quality as tangential slicing direction is approached. Apart from these limitations, further general problems exist that hinder a wide dissemination of virtual endoscopy of the intestinal tract as a primary population

T. Dohi and R. Kikinis (Eds.): MICCAI 2002, LNCS 2489, pp. 20-27 2002.

(C) Springer-Verlag Berlin Heidelberg 2002 
screening procedure. These include the relatively lengthy time required for data interpretation, poor patient compliance regarding bowel cleansing, and concerns over the $\mathrm{CT}$ radiation dose.

Our current research is directed at solving these problems by using MR imaging for virtual endoscopy of the intestinal tract - especially the small bowel. To improve patient compliance, we propose a new concept with an oral approach to avoid the need of invasive intubation which is more acceptable to the patient. Furthermore, we enhance the image analysis process by interactive haptic segmentation methods.

\section{Medical Background}

The prevalence of small bowel disease - the most common being Crohn's Disease (chronic inflammatory bowel disease) and small bowel carcinoid tumor or tumor metastasis - is low, and the clinical diagnosis is complicated by nonspecific symptoms and a low index of suspicion. This frequently leads to delays in diagnosis and treatment. An accurate radiologic examination is, therefore, important not only for recognition of small bowel disease but also to help reliably document normal morphology [8]. The limitations of conventional enteroclysis (small bowel barium contrast x-ray) investigation, which needs invasive nasoduodenal intubation for contrast material application, have been recognized for a long time $[4]$.



(a) $2 \mathrm{D}$ slice view.

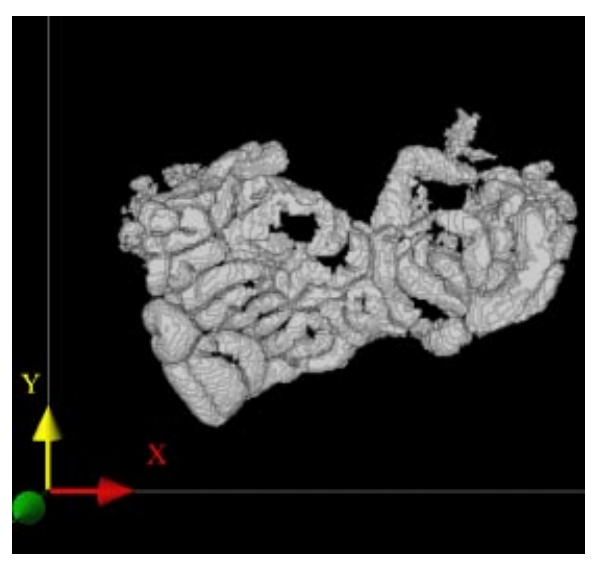

(b) Thresholded 3D view.

Fig. 1. Small intestine image data.

Despite advances in fibre-optic endoscopy, the majority of the small bowel still remains inaccessible. Although recently developed small endoscopes allow true endoscopy of the duodenum and proximal jejunum, the conventional and cross-sectional gastroenterologic imaging methods currently represent the only reliable technique for evaluating the small bowel. The functional information, 
soft-tissue contrast, direct multiplanar capabilities, and lack of ionizing radiation suggest that MR imaging has a greater potential than other techniques to become the ideal diagnostic method for imaging of the small bowel. After acquisition of the volumetric data with an MR, the mesenteric small bowel has to be assessed by a radiologist. However, doing this step on cross sectional images is somewhat analogous to separating out and identifying a large number of writhing snakes in a crowded reptile tank. A more promising approach is using virtual endoscopy techniques, which have been a major research focus in recent years. The creation of three-dimensional images with perspective distortion promises also for the small bowel to be an advancement in diagnostics. Nevertheless, virtual endoscopy of the small bowel is much more difficult than virtual colonoscopy and can not yet be performed by the currently available postprocessing tools. The manual path definition proves difficult in the sharp turns of the small bowel and the loss of orientation is the most obvious problem (Figure 1). The most crucial task for future integration of small bowel examination into clinical routine is the development of more reliable segmentation tools and path finding systems for virtual endoscopy of the small intestine. As a consequence, we aim at enhancing this process with a new interactive haptic tool for segmentation and centerline definition.

\section{Image Acquisition}

Driven by public concern about medical radiation exposure, we developed a robust, albeit complex, technique for high-quality MR imaging [11,7]. Prior to MR imaging of the small bowel, patients were prepared by oral ingestion of four doses of stool softener spiked with a clinically used MR contrast agent starting three hours before the examination. This mixture forms a viscous hydrogel within the intestinal lumen, giving good luminal distension, constant signal homogeneity, sufficient demarcation of the bowel content from surrounding tissues, and a low rate of artifacts, thus permitting non-invasive high quality MRI of the small bowel. According to the report of three volunteers and twelve patients, the oral mixture was well tolerated apart from slight abdominal discomfort and a sensation of being full. Data acquisition was performed breathhold in coronal plane, with the patient in a prone position. This near isotropic volume acquisition strategy permits multiplanar and three-dimensional reconstructions. Because MR imaging remains a motion-sensitive technique, bowel peristalsis is reduced by intravenous administration of a spasmolytic drug. The availability of high-performance gradient systems allows for the acquisition of large data volumes within a single breathhold [6], thereby eliminating respiratory motion artifacts. To assure data acquisition in apnea, imaging times are maintained under 30 seconds, limiting the number of contiguous $2 \mathrm{~mm}$ sections to $48-64$. The technique is based on the use of very short echo and repetition times rendering most tissues, including fat, dark. The signal is evident only within regions containing T1-shortening contrast in a concentration sufficient to reduce $\mathrm{T} 1$ relaxation times to levels below 50ms [9]. 


\section{Interactive Segmentation System}

After acquiring the image data of the small intestines, the data sets have to be segmented into their major structural components before any high-level reasoning can be applied. As a consequence of the complex, tightly packed geometry of the small bowel, up to now no method is available, which could reliably provide a topologically correct segmentation. Even manual identification of the organ outline on $2 \mathrm{D}$ slices, which is usually the last rescue in case of lacking other alternatives, proved to be inappropriate due to the difficulties discussed in the introduction. Therefore, we had to apply a new virtual reality-based interaction metaphor for semi-automatic segmentation of medical $3 \mathrm{D}$ volume data $[2$, 3]. The mouse-based, manual initialization of deformable surfaces in 3D represents a major bottleneck in interactive segmentation. In our multi-modal system we enhance this process with additional sensory feedback. A 3D haptic device is used to extract the centerline of a tubular structure. Based on the obtained path a cylinder with varying diameter is generated, which in turn is used as the initial guess for a deformable surface. In the following sections we will describe our approach in detail.

\subsection{Data Preparation}

The initial step of our multi-modal approach is the haptically assisted extraction of the centerline of a tubular structure. First we create a binarization of our data volume by thresholding. We have to emphasize that this step is not sufficient for a complete segmentation of the datasets we are interested in. This is due to the often low quality of the image data caused by unevenly distributed contrast agents, pathological changes and partial volume effects. Nevertheless, in the initial step we are not interested in a topologically correct segmentation. On the contrary, we only need a rough approximation of our object of interest. For each voxel that is part of the tubular structure we compute the Euclidean distance to a voxel of the surrounding tissue. In the next step we negate the 3D distance map and approximate the gradients by central differences. Moreover, to ensure the smoothness of the computed forces, we apply a $5 \times 5 \times 5$ binomial filter. This force map is precomputed before the actual interaction to ensure a stable force-update. Because the forces vectors are located at discrete voxel positions, we have to do a tri-linear interpolation to obtain the continuous gradient force map needed for stable haptic interaction. Furthermore, we apply a low-pass filter in time to further suppress instabilities.

\subsection{Centerline Extraction}

The goal of the centerline extraction process is to identify the ridge line through the resulting distance map. As in most object identification tasks the basic problem is to ensure the connectivity of the result by closing the gaps through areas, where the ridge is less pronounced. Haptic feedback proved to be a very efficient and intuitive metaphor to solve this problem. In the optimal case of good data quality, the user "falls through" the data set guided along the 3D ridge created by the forces. While moving along the path, control points are set, which are 
used to approximate the path with a spline. At regions with less clear image information, an expert can use his knowledge to guide the 3D cursor through fuzzy or ambiguously defined areas by exerting force on the haptic device to actively support path definition.

\subsection{Segmentation}

The next step is to use the extracted centerline to generate a good initialization for a deformable surface model. To do this, we create a tube around the path with varying thickness according to the precomputed distance map. This object is then deformed subject to a thin plate under tension model. Assuming positionindependent damping and homogeneous material properties as well as using discrete approximations of the differential operators, we can use Gauss-Seidel iteration to solve the resulting system of Euler-Lagrange equations.

$$
\gamma \mathbf{v}_{t}-\tau \Delta \mathbf{v}+(1-\tau) \Delta^{2} \mathbf{v}=-\frac{\delta P}{\delta \mathbf{v}}
$$

Due to the good initialization, only a few steps are needed to approximate the desired object. The path initialization can be seen in Figure 2(a). Note, that the $3 \mathrm{D}$ data is rendered semi-transparent to visualize the path in the lower left portion of the data. Figure 2(b) depicts the surface model during deformation.

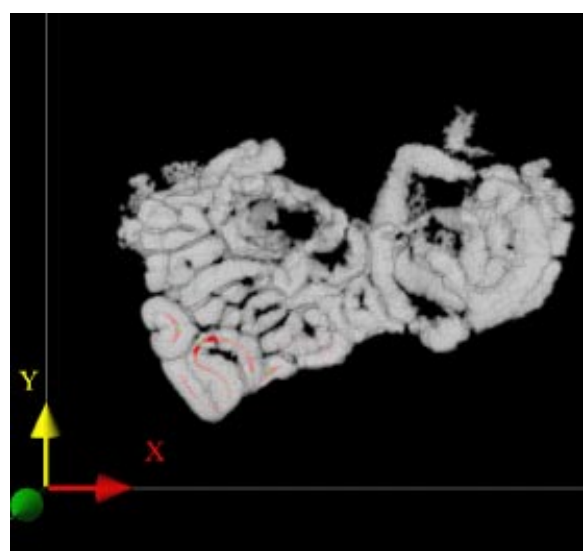

(a) Initialized path.

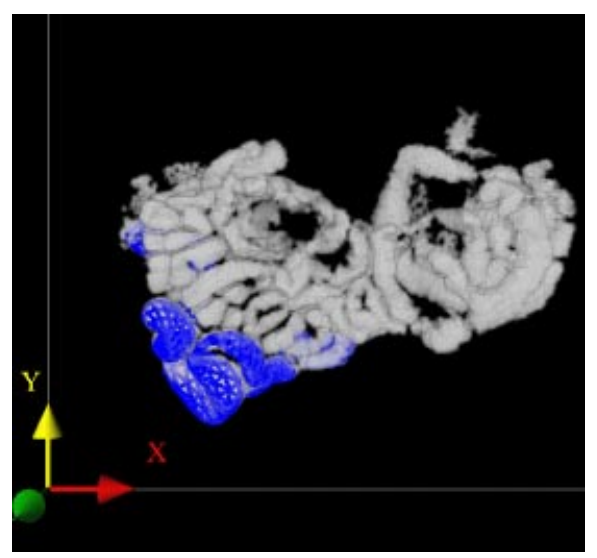

(b) Deforming tube.

Fig. 2. Interactive Segmentation.

In order to further improve the interaction with complicated data sets we adopt a step-by-step segmentation approach by hiding already segmented loops. This allows a user to focus his attention on the parts that still have to be extracted. For this purpose we have to turn the 3D surface model back into voxels, which should happen fast enough to maintain real time interaction. To achieve this goal we make use of the graphics hardware by implementing a zbuffer based approach as described in [5]. This process is shown in Figure 3. 


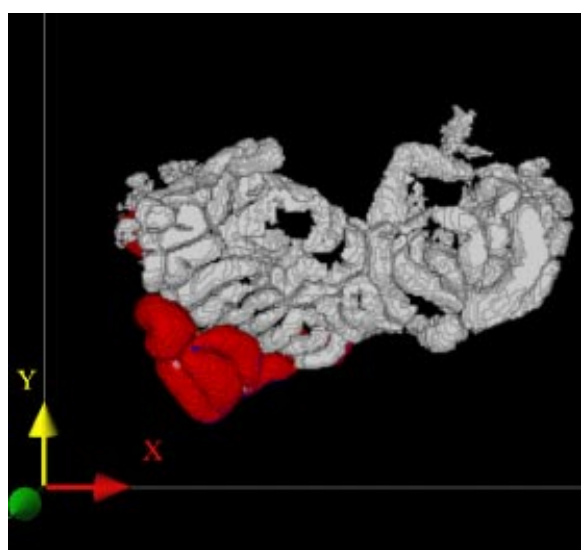

(a) Voxelization.

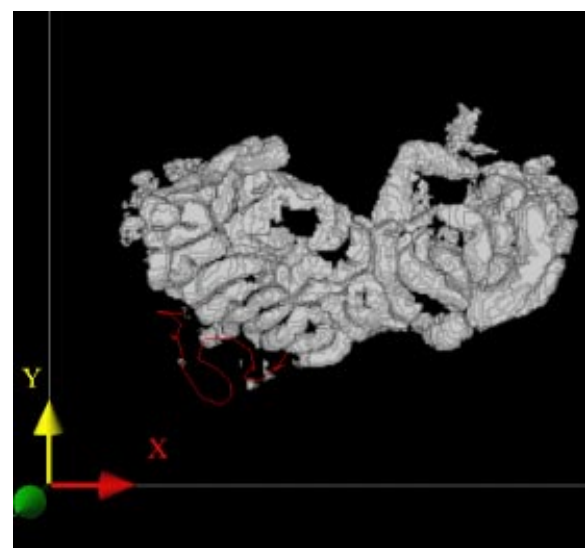

(b) Removed segmented part.

Fig. 3. Hiding segmented parts.

\subsection{System Evaluation}

We carried out an initial test study to evaluate the influence of haptic interaction on the performance of centerline extraction and the following segmentation. The experiment followed a within-subjects repeated-measures design. Five participants took part in the study, only one had used a haptic device before.

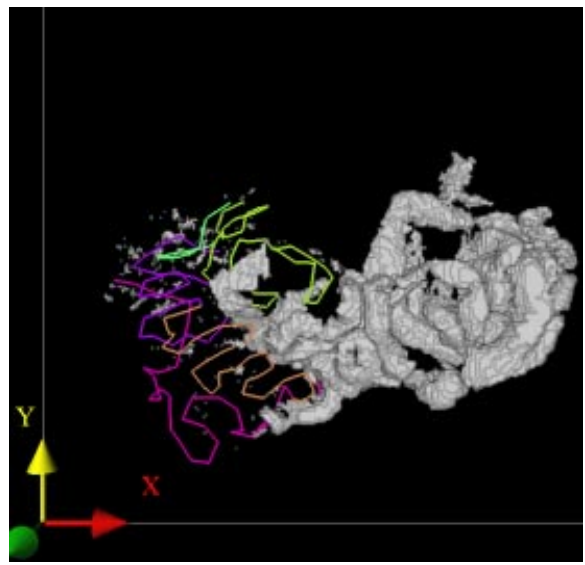

(a) Start of process.

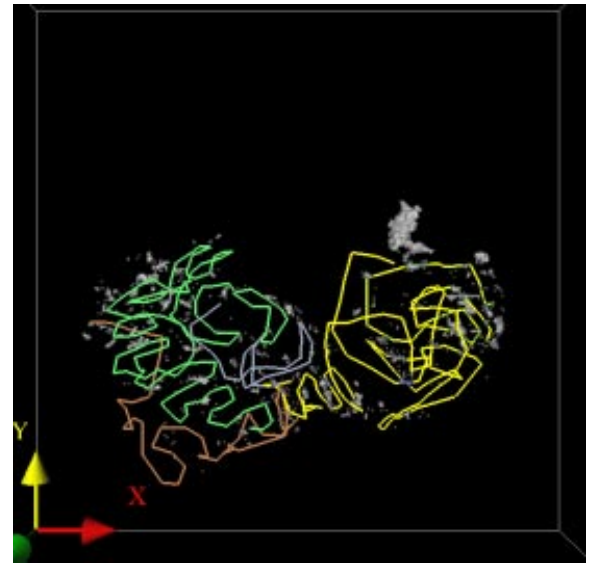

(b) Complete extraction.

Fig. 4. Centerline extraction.

Subjects were introduced to the interactive segmentation tool and were informed how to set a centerline with the system. Also, to familiarize the subjects with force-feedback, we presented them haptic rendering of the surface of the 
voxel objects based on data gradients. Each subject carried out the experiment under two conditions, without and with haptic enhancement for centerline tracing. The segmentation task was performed on an artificial and a real data set. The performance measure was the interaction time in seconds for model initialization. After setting the path, a 3D deformable surface was initialized and deformed without user interaction. The effect of the path quality on the segmentation process was also examined in our study. The data samples taken are paired allowing an analysis of difference to be undertaken. The distributions were successfully tested for normality, thus allowing the use of a paired t-test. A summary of the acquired data is shown in Table 1. This initial study has shown that there is a statistically significant performance improvement in the trial time $(t=3.59, d f=9, p \leq 0.007)$ when using haptically enhanced interaction in $3 \mathrm{D}$ segmentation. Also in the haptic condition the quality of segmentation was always superior to the one without force-feedback. In seven out of ten cases, the deformable surface, initialized based only on visual feedback, collapsed in parts of the structure, thus requiring additional user interaction. This is due to imprecise initialization of the centerline, which causes the deformable model in poorly initialized regions to fail to automatically extract the object of interest. Subjects reported that 3D positioning was substantially facilitated with the force-feedback. Moreover, although most of the participants expressed a need for longer training in haptic interaction itself, all of them were already successful in taking advantage of the technology. Generally, subjects stated that in the haptically assisted condition they mainly focused on using the forces for guidance, while the visual feedback was only used for fine-tuning.

\begin{tabular}{|l||c|c|}
\hline \multicolumn{3}{|c|}{ Initialization time. } \\
\hline \hline & Visual only & With haptics \\
\hline Mean trial time (s) & 147.0 & 73.1 \\
\hline Standard deviation & 85.6 & 37.5 \\
\hline
\end{tabular}

Table 1. Results of initial study.

\section{Results}

Three healthy volunteers (without any history of gastrointestinal disease or surgery) and twelve patients (evaluation of small bowel obstruction or chronic inflammatory bowel disease) participated in this preliminary study. We were able to use our system to obtain the centerline through the small intestines. Figure 4(a) shows the start of the process and Figure 4(b) displays the final outcome. Please note, that some of the shorter sections in the first image were combined into longer ones.

\section{Conclusions}

We have shown a new approach to generating computer models for virtual endoscopy based on MR image acquisition and haptically enhanced interactive 
segmentation. We acquired high-quality images of the small intestines and used our system to completely segment the small bowel - to the best of our knowledge, this has not been achieved before. Whether our approach will readily replace currently used methods of small bowel imaging will depend on how this method can be integrated into the clinical setting in a practical manner that will be acceptable to patients, referring clinicians, and surgeons. To be the primary method for investigation of small-bowel disease, MR imaging will have to provide reliable evidence of normalcy, allow diagnosis of early or subtle structural abnormalities and influence treatment decisions in patient care. Further research and experience will help clarify whether our approach should be the primary method for investigation of the small bowel or used only as a problem-solving examination. Preliminary clinical investigation using the described system have given rise to the following recommended improvements: increased spatial and temporal resolution in MR imaging of small bowel to achieve true isotropic imaging, to assess ideal timing between intake of oral contrast agent and imaging, optimization of small bowel distension and further refinement of the current tools for segmentation and path definition. Nevertheless, the developed imaging, segmentation and navigation methods already opened a way for the extension of virtual endoscopy investigations onto the whole intestinal tract.

\section{Acknowledgment}

This work has been performed within the frames of the Swiss National Center of Competence for Research in Computer Aided and Image Guided Medical Interventions (NCCR CO-ME) supported by the Swiss National Science Foundation.

\section{References}

1. H.M. Fenlon, D.P. Nunes, P.C. Schroy, M.A. Barish, P.D. Clarke, and J.T. Ferrucci. A comparison of virtual colonoscopy and conventional colonoscopy for the detection of colorectal polyps. In N Engl J Med, pages 1496-1503, 1999.

2. M. Harders and G. Székely. Improving medical segmentation with haptic interaction. In IEEE Computer Society Conf. on Virtual Reality, 2002.

3. M. Harders and G. Székely. New paradigms for interactive 3d volume segmentation. In Journal of Visualization and Computer Animation, 2002.

4. H. Herlinger and D.D.T. Maglinte. Clinical radiology of the small intestine, 1989. 41-44.

5. E.-A. Karabassi, G. Papaioannou, and T. Theoharis. A fast depth-buffer-based voxelization algorithm. Journal of Graphics Tools, 4(4):5-10, 1999.

6. D.A. Leung, G.C. McKinnon, C.P. Davis, T. Pfammatter, G.P. Krestin, and J.F. Debatin. Breathheld contrast-enhanced 3d mr angiography. In Radiology, 1996.

7. W. Luboldt, P. Bauerfeind, S. Wildermuth, B. Marincek, M. Fried, and J.F. Debatin. Colonic masses: detection with mr colonography. In Radiology, 2000.

8. D.D.T. Maglinte, K. O'Connor, J. Bessette, S.M. Gernish, and F.M. Kelvin. The role of physician in the late diagnosis of primary malignant tumors of the small intestine. American Journal of Gastroenterology, 86:304-308, 1991.

9. M.R. Prince. Gadolinium-enhanced mr aortography. In Radiology, 1994.

10. D.J. Vining. Virtual endoscopy: Is it. In Radiology, pages 30-31, 1996.

11. S. Wildermuth and J.F. Debatin. Virtual endoscopy in abdominal mr imaging. In Magn Reson Imaging Clin N Am., pages 349-364, 1999. 